\title{
Spin-flip transitions between Zeeman sublevels in semiconductor quantum dots
}

\author{
Alexander V. Khaetskii* and Yuli V. Nazarov \\ Faculty of Applied Sciences and DIMES, Delft University of Technology, Lorentzweg 1, 2628 CJ Delft, The Netherlands
}

(Received 26 January 2001; published 10 September 2001)

\begin{abstract}
We have studied spin-flip transitions between Zeeman sublevels in GaAs electron quantum dots. Several different mechanisms which originate from spin-orbit coupling are shown to be responsible for such processes. It is shown that spin-lattice relaxation for the electron localized in a quantum dot is much less effective than for the free electron. The spin-flip rates due to several other mechanisms not related to the spin-orbit interaction are also estimated.
\end{abstract}

DOI: 10.1103/PhysRevB.64.125316

PACS number(s): 85.30.De, 71.70.Ej, 73.23.-b

\section{INTRODUCTION}

Quantum dots (QD's) in semiconductor heterostructures provide a unique opportunity to study the properties of the electron quantum states in detail and manipulate the electrons in these "artificial atoms" in a controllable way (see reviews in Refs. 1 and 2). The shape and size of quantum dots can be varied by changing the gate voltage. This also tunes the number of electrons in the dot. Besides, the electronic states can be significantly modified by a magnetic field applied perpendicular to the plane of the heterostructure.

Quantum dots are considered as possible candidates for building a quantum computer. ${ }^{3}$ The crucial point of the idea is the necessity to couple dots coherently and keep coherence on sufficiently long time scales. In this respect, there is a great demand in the theoretical estimation of the typical spin dephasing time of the electron in the QD. In our previous work ${ }^{4}$ we have shown that the localized character of the electron wave functions in the QD's suppresses the most effective intrinsic spin-flip mechanisms related to the absence of inversion symmetry in GaAs-like crystals. This leads to an unusually low rate of spin-flip transitions. However, in Ref. 4 we concentrated on the case of inelastic transitions between the neighboring quantized energy levels in the dot which corresponds to a relatively large energy transfer. On the other hand, the quantum bit was proposed to involve two Zeeman sublevels of the same orbital level. Therefore, in the present work we consider the transitions between such sublevels. Since the transition involves a fairly small energy transfer, the results are very different from those of Ref. 4.

As in Ref. 4, we concentrate on the spin-flip processes due to the spin-orbit interaction. This is the main source of the spin flips for the three- and two-dimensional (3D and 2D) electron states in the GaAs-type crystal without an inversion center. Besides, in such a polar-type crystal one finds a strong coupling of electrons to the bosonic environment via the piezoelectric interaction with acoustic phonons. The combination of these two mechanisms provides an effective spin-lattice relaxation of free carriers in $A_{I I I} B_{V}$ semiconductors and heterostructures. ${ }^{5}$ We show, however, that the spinlattice relaxation for the electron localized in the QD is much less effective.

We have calculated the rates for the different spin-orbitrelated mechanisms which cause a spin flip in the course of the phonon-assistant transition between the Zeeman sublev- els. Besides, we have estimated the spin-flip rates due to several other mechanisms, for example, due to the fluctuating magnetic field produced by the fluctuating electron density in the leads or due to modulation of the hyperfine coupling with nuclei by lattice vibrations.

\section{SPIN-ORBIT MECHANISMS}

We consider the case of a strong confinement in the $z$ direction and the typical lateral dot size is of the order of $1000 \AA$ and much larger than the width of the 2D layer in the $z$ direction. We begin with the following one-electron Hamiltonian that is derived from the Kane model (see Ref. 5) and describes the 2D electrons of the conduction band in the presence of the magnetic field $\mathbf{B}$, lateral confining potential $U(\mathbf{r})$, and phonons:

$$
\begin{gathered}
\hat{\mathcal{H}}=\frac{\hat{\mathbf{p}}^{2}}{2 m}+U(\mathbf{r})+U_{p h}(\mathbf{r}, t)+\frac{1}{2} g \mu_{B} \hat{\boldsymbol{\sigma}} \cdot \mathbf{B}+\sum_{i=1}^{3} \hat{\mathcal{H}}_{i}, \\
\hat{\mathcal{H}}_{1}=\beta\left(-\hat{\sigma}_{x} \hat{p}_{x}+\hat{\sigma}_{y} \hat{p}_{y}\right) ; \beta=\frac{2}{3}\left\langle p_{z}^{2}\right\rangle \frac{\Delta}{\left(2 m E_{g}\right)^{1 / 2} m_{c v} E_{g}}, \\
\hat{\mathcal{H}}_{2}=\frac{1}{2} V_{0} \hat{\boldsymbol{\sigma}} \cdot \hat{\boldsymbol{\varphi}} ; \quad \hat{\mathcal{H}}_{3}=\tilde{g} \mu_{B} \sum_{i \neq k} u_{i k} \hat{\sigma}_{i} B_{k} .
\end{gathered}
$$

Here $\hat{\mathbf{p}}=-i \hbar \boldsymbol{\nabla}+(e / c) \mathbf{A}$ is the $2 \mathrm{D}$ electron momentum operator, $m$ the effective mass, and $\hat{\boldsymbol{\sigma}}$ the Pauli matrices. The axes $x, y, z$ coincide with the main crystallographic ones with the $z$ axis along the normal to the 2D plane (the [100] orientation). The magnetic field has an arbitrary direction. The third term describes the spin-independent interaction with the phonons, including the piezoelectric ones. The fourth term is the Zeeman energy. The other three terms describe all possible spin-orbit effects. $\hat{\mathcal{H}}_{1}$ stems from the absence of the inversion symmetry in the bulk. ${ }^{6}$ Velocity $\beta$ takes the values in the interval $(1-3) \times 10^{5} \mathrm{~cm} / \mathrm{s}$ for GaAs heterostructures. $\hat{\mathcal{H}}_{2}$ describes the spin-orbit splitting of the electron spectrum due to the strain field produced by the acoustic phonons. There $\hat{\varphi}_{x}=(1 / 2)\left\{u_{x y}, \hat{p}_{y}\right\}_{+}, \quad \hat{\varphi}_{y}=-(1 / 2)\left\{u_{y x}, \hat{p}_{x}\right\}_{+}$, and $\hat{\varphi}_{z}=(1 / 2)\left\{u_{z x}, \hat{p}_{x}\right\}_{+}-(1 / 2)\left\{u_{z y}, \hat{p}_{y}\right\}_{+}$, where $\{,\}_{+}$denotes the anticommutator, $u_{i j}$ is the lattice strain tensor, and $V_{0}$ $=8 \times 10^{7} \mathrm{~cm} / \mathrm{s}^{7}$ In GaAs the electron $g$ factor $(g=-0.44)$ 
differs strongly from the free electron value $g_{0}=2$ owing to the strong spin-orbit interaction which mixes the valenceband and conduction-band states. ${ }^{8}$ The admixture depends on the lattice deformation. The coefficient $\tilde{g}$ can be found within the Kane approach, $\tilde{g}=\left(2 m_{0} / \sqrt{3} m\right)\left(\Delta / E_{g}\right)\left(d / E_{g}\right)$; $d=-4.5 \mathrm{eV}$ is one of the three deformation constants describing the strain effect on the hole-band splitting, ${ }^{5} \tilde{g}$ $\approx 10.4$.

The three terms in Hamiltonian (1) correspond to the three distinct mechanisms of the spin flip. The first mechanism is due to the spin-orbit admixture of the state with an opposite spin. While without the spin-orbit interaction the Zeeman sublevels correspond to the orbital state with the spin up or down, the spin-orbit terms provide a small admixture of the state of the opposite spin to each sublevel. This enables the phonon-assistant transition between the two states. This mechanism corresponds to term $\hat{\mathcal{H}}_{1}$.

The second and third mechanisms are described by the $\hat{\mathcal{H}}_{2}$ and $\hat{\mathcal{H}}_{3}$ terms and correspond to two distinct kinds of direct spin-phonon coupling. Below we show that the admixture mechanism is actually a dominant one.

\section{A. Admixture mechanism}

Let us show that for this mechanism the matrix element of the $U_{p h}(\mathbf{r}, t)$ operator for the spin-flip transition between the Zeeman levels is proportional to the product of the Zeeman energy and the phonon strain field. Since we deal with a small energy transfer, we consider only the interaction with piezophonons; hence, for mode $\mathbf{q} \alpha, \alpha=l, t$, we have ${ }^{9}$

$$
\begin{gathered}
U_{p h}^{\mathbf{q} \alpha}(\mathbf{r}, t)=\sqrt{\hbar / 2 \rho \omega_{\mathbf{q} \alpha}} \exp \left(i \mathbf{q r}-i \omega_{\mathbf{q} \alpha} t\right) e A_{\mathbf{q} \alpha} b_{\mathbf{q} \alpha}^{+}+\text {c.c. } \\
A_{\mathbf{q} \alpha}=\xi_{i} \xi_{k} \beta_{i k j} e_{\mathbf{q} \alpha}^{j}
\end{gathered}
$$

where the effective piezoelectric modulus $A_{\mathbf{q} \alpha}$ of wave $\mathbf{q} \alpha$ has been introduced, $\beta_{i k j}$ the piezotensor, $\boldsymbol{\xi}=\mathbf{q} / q$, $\mathbf{q}$ the phonon wave vector, $\mathbf{e}$ the phonon unit polarization vector, and $\rho$ the crystal mass density. For the crystal of cubic symmetry without an inversion center (class $T_{d}$ ) the tensor $\beta_{i k j}$ has only nonzero components (all of them equal to each other) when all three indexes $i, k, j$ are different, $\beta_{x y z}=\beta_{x z y}=\ldots$. $=h_{14}$. For GaAs, $e h_{14}=1.2 \times 10^{7} \mathrm{eV} / \mathrm{cm}$; see, for example, Ref. 9.

The matrix element for the spin-flip transition between the Zeeman sublevels of orbital level $n$ with emission of phonon $\mathbf{q} \alpha$ is

$$
\left\langle n \uparrow\left|U_{p h}^{\mathbf{q} \alpha}\right| n \downarrow\right\rangle=\sum_{k \neq n}\left[\frac{\left(U_{p h}^{\mathbf{q} \alpha}\right)_{n k}\left(\hat{\mathcal{H}}_{1}\right)_{k n}^{\uparrow \downarrow}}{E_{n}-E_{k}-g \mu_{B} B}+\frac{\left(\hat{\mathcal{H}}_{1}\right)_{n k}^{\uparrow \downarrow}\left(U_{p h}^{\mathbf{q} \alpha}\right)_{k n}}{E_{n}-E_{k}+g \mu_{B} B}\right],
$$

where the states $n, k$ and corresponding energies $E_{n}, E_{k}$ are determined by first two terms in Hamiltonian (1). The spin quantization axis coincides with the magnetic field vector. In the absence of a magnetic field the two terms in Eq. (3) cancel each other since $\left(\hat{\mathcal{H}}_{1}\right)_{n k}^{\uparrow \downarrow}=-\left(\hat{\mathcal{H}}_{1}\right)_{k n}^{\uparrow \downarrow}$ and the matrix elements involving the phonon operator are symmetric with respect to the interchange of indexes $n$ and $k$. This "Van Vleck cancellation" 10,11 is a consequence of Kramers' theorem and reduces the matrix element by a factor of $g \mu_{B} B / \hbar \omega_{0}, \hbar \omega_{0}$ being the typical distance between the orbital levels in the dot. Note that this cancellation occurs for a spin-orbit Hamiltonian of an arbitrary form. For instance, it could include the third-order terms in the lateral momentum operator. ${ }^{4}$ This is in strong contrast with the cancellation of the linear in the $\beta$ terms in the matrix elements for the spinflip transition between different orbital levels, ${ }^{4}$ which results from the fact that spin-orbit terms $\hat{\mathcal{H}}_{1}$ are linear in the lateral momentum operators $\hat{p}_{x, y}$. Expanding in the above formula with respect to the Zeeman energy, using the relation $\left(\hat{p}_{i}\right)_{n k}=(i m / \hbar)\left(E_{n}-E_{k}\right)\left(x_{i}\right)_{n k}$ and the condition that the phonon wavelength be much larger than the dot size (i.e., $g \mu_{B} B \ll \sqrt{m s^{2} \hbar \omega_{0}} ; s$ is the sound velocity), we obtain the efective spin-flip Hamiltonian which acts on the subspace of the Zeeman sublevels of orbital level $n$ :

$$
\begin{aligned}
\hat{H}_{s o}^{(n)}= & g \mu_{B} B \frac{m \beta}{\hbar e}\left[\hat{\sigma}_{x} \alpha_{x x}^{(n)} E_{x}-\hat{\sigma}_{y} \alpha_{y y}^{(n)} E_{y}\right. \\
& \left.+\frac{\left(\alpha_{x y}^{(n)}+\alpha_{y x}^{(n)}\right)}{2}\left(\hat{\sigma}_{x} E_{y}-\hat{\sigma}_{y} E_{x}\right)\right],
\end{aligned}
$$

where $E_{x, y}=-\nabla_{x, y} U_{p h}(x, y) / e$ is the phonon-induced electric field in the location of the dot. Here we introduce the polarizability tensor $\hat{\alpha}$ that may depend on $B_{z}$. It is given by

$$
\alpha_{i k}^{(n)}\left(B_{z}\right)=-2 e^{2} \sum_{m \neq n} \frac{\left(x_{i}\right)_{n m}\left(x_{k}\right)_{m n}}{E_{n}-E_{m}} .
$$

The effective Hamiltonian (4) is a very general one and can be used to calculate the spin-flip rates for arbitrary states and dots. To specify, we will consider only parabolic elliptic dots with the main axes along the $x, y$ symmetry axes, $\omega_{x, y}$ being the oscillator frequencies. Then the symmetry of the kinetic coefficients ensures that $\left(\alpha_{x y}^{(n)}+\alpha_{y x}^{(n)}\right)=0$. We have to calculate the spin-flip matrix elements $\left\langle+1 / 2\left|\hat{\sigma}_{x, y}\right|-1 / 2\right\rangle$ over the functions $\Psi_{\mu}, \mu= \pm 1 / 2$, which are the eigenfunctions of operator $\hat{\sigma}_{z^{\prime}}$, where the $z^{\prime}$ axis is directed along the magnetic field vector. These functions are expressed through the eigenfunctions $\chi_{m}$ of $\hat{\sigma}_{z}$ operator: $\Psi_{\mu}=\sum_{m= \pm 1 / 2} D_{\mu m}^{(1 / 2) \star}$ $\times(\varphi, \vartheta, 0) \chi_{m}$, where $D^{(1 / 2)}$ is the finite rotation matrix ${ }^{12}$ and $\varphi, \vartheta$ are the azimuthal and polar angles presenting $\mathbf{B}$ in the spherical coordinates. We substitute $E_{x, y}$ in terms of the boson creation and annihilation operators. Then for the square modulus of the spin-flip matrix element that involves the emission of a phonon with wave vector $\mathbf{q}$ we obtain

$$
\begin{aligned}
\left|\hat{H}_{s o}^{\uparrow \downarrow}(\mathbf{q} \alpha)\right|^{2}= & \left(\frac{g \mu_{B} B m \beta}{\hbar e}\right)^{2} A_{\mathbf{q} \alpha}^{2}\left(\frac{\hbar}{2 \rho \omega_{\mathbf{q} \alpha}}\right)\left\{\left(\alpha_{x x}^{2} q_{x}^{2}\right.\right. \\
& \left.+\alpha_{y y}^{2} q_{y}^{2}\right) \frac{\left(1+\cos ^{2} \vartheta\right)}{2}-\frac{\sin ^{2} \vartheta}{2}\left[\left(\alpha_{x x}^{2} q_{x}^{2}\right.\right. \\
& \left.\left.\left.-\alpha_{y y}^{2} q_{y}^{2}\right) \cos 2 \varphi-2 \alpha_{x x} \alpha_{y y} q_{x} q_{y} \sin 2 \varphi\right]\right\} .
\end{aligned}
$$


The summing up over all $\mathbf{q}$ yields the rate due to the first mechanism:

$$
\begin{aligned}
\Gamma_{1}= & \frac{2 \pi}{\hbar} \int \frac{d^{3} q}{(2 \pi)^{3}} \sum_{\alpha=l, t} C_{\alpha}\left|\hat{H}_{s o}^{\uparrow \downarrow}(\mathbf{q} \alpha)\right|^{2} \delta\left(\hbar s_{\alpha} q-g \mu_{B} B\right) \\
= & \frac{\left(g \mu_{B} B\right)^{5}}{35 \pi \rho \hbar^{4}}\left(\frac{h_{14} m \beta}{e \hbar}\right)^{2}\left[\left(\alpha_{x x}^{2}+\alpha_{y y}^{2}\right)\left(1+\cos ^{2} \vartheta\right)\right. \\
& \left.-\left(\alpha_{x x}^{2}-\alpha_{y y}^{2}\right) \sin ^{2} \vartheta \cos 2 \varphi\right]\left(\frac{1}{s_{l}^{5}}+\frac{4}{3 s_{t}^{5}}\right) .
\end{aligned}
$$

Here $C_{l}=1, C_{t}=2$ and $s_{l}, s_{t}$ are the longitudinal and transverse sound velocities. The anisotropy factors used are $A_{\mathbf{q}, l}^{2}=36 h_{14}^{2} \cos ^{2} \theta \sin ^{4} \theta \sin ^{2} \phi \cos ^{2} \phi$ where $\phi$ and $\theta$ are the azimuthal and polar angles of vector q. Here $\left\langle A_{\mathbf{q}, t}^{2}\right\rangle=4 h_{14}^{2}\left\langle\left(\xi_{x} \xi_{y} e_{z}+\xi_{x} \xi_{z} e_{y}+\xi_{y} \xi_{z} e_{x}\right)^{2}\right\rangle=2 h_{14}^{2}\left[\cos ^{2} \theta \sin ^{2} \theta\right.$ $\left.+\sin ^{4} \theta\left(1-9 \cos ^{2} \theta\right) \sin ^{2} \phi \cos ^{2} \phi\right]$, where $\langle\cdots\rangle$ means averaging over the orientations of the e vector in the plane which is perpendicular to $\mathbf{q}$. The averaging is done by the formula $\left\langle e_{i} e_{k}\right\rangle=(1 / 2)\left(\delta_{i k}-\xi_{i} \xi_{k}\right)$. As usual, in the case of finite temperature Eq. (7) should be multiplied by factors $N_{\omega}$ $+1\left(N_{\omega}\right)$ for the transition with emission (absorption) of a phonon, $N_{\omega}=1 /\left(e^{\hbar \omega / T}-1\right), \hbar \omega=g \mu_{B} B$. Thus, in the case of high temperature $T \gg g \mu_{B} B$ the spin-flip rate will be proportional to $\left(g \mu_{B} B\right)^{4} T$.

In the particular case of a circular dot $\omega_{x}=\omega_{y}=\omega_{0}$ we have $\alpha_{x x}\left(B_{z}\right)=\alpha_{y y}\left(B_{z}\right)=\alpha_{x x}(0)=e^{2} / m \omega_{0}^{2}$. Then, for instance, for the transition between the Zeeman sublevels of the ground state of the circular dot with emission of a piezophonon we obtain

$$
\begin{gathered}
\Gamma_{1}=\frac{\left(g \mu_{B} B\right)^{5}}{\hbar\left(\hbar \omega_{0}\right)^{4}} \Lambda_{p}\left(1+\cos ^{2} \vartheta\right), \\
\Lambda_{p} \equiv \frac{2}{35 \pi} \frac{\left(e h_{14}\right)^{2} \beta^{2}}{\rho \hbar}\left(\frac{1}{s_{l}^{5}}+\frac{4}{3 s_{t}^{5}}\right) .
\end{gathered}
$$

The dimensionless constant $\Lambda_{p}$ shows the strength of the effective spin-piezophonon coupling in the heterostructure and ranges from $\approx 7 \times 10^{-3}$ to $\approx 6 \times 10^{-2}$ depending on $\beta$. The spin-flip rate exhibits a very strong dependence on the Zeeman energy and lateral confinement energy $\omega_{0}$. To give a number, $\Gamma_{1} \approx 1.5 \times 10^{3} \mathrm{~s}^{-1}$ for $\hbar \omega_{0}=10 \mathrm{~K}$ and a relatively large magnetic field $B=1$ T.

Formula (3) is written with allowance for the wave function corrections of the first order with respect to the spinorbit Hamiltonian. The corrections of the second order are described by the following spin-orbit Hamiltonian:

$$
\hat{\mathcal{H}}_{\sigma_{z}}=\frac{m \beta^{2}}{\hbar} \hat{\sigma}_{z}\left(x \hat{p}_{y}-y \hat{p}_{x}\right) .
$$

Then, using this Hamiltonian in formula (3) instead of $\hat{\mathcal{H}}_{1}$, we can get a nonzero contribution to the spin-flip matrix element even with zero Zeeman splitting in the denominator (but with taking into account the orbital magnetic field). Keeping again only the term which is linear in $\mathbf{q} \cdot \mathbf{r}$ in the expansion of exponent $\exp (i \mathbf{q} \cdot \mathbf{r})$, for the rate finally we obtain

$$
\begin{aligned}
\Gamma_{\uparrow, \downarrow}^{(n)}= & \frac{2}{35 \pi} \frac{\left(g \mu_{B} B\right)^{3}\left(e h_{14}\right)^{2}}{\hbar^{4} \rho}\left(\frac{m \beta^{2}}{\hbar}\right)^{2} \sin ^{2} \vartheta\left[\left(D_{x}^{(n)}\right)^{2}\right. \\
& \left.+\left(D_{y}^{(n)}\right)^{2}\right]\left(\frac{1}{s_{l}^{5}}+\frac{4}{3 s_{t}^{5}}\right),
\end{aligned}
$$

where

$$
\begin{gathered}
D_{x}^{(n)}=2 \operatorname{Re} \sum_{m \neq n} \frac{x_{n m}\left[\hat{L}_{z}+\left(e B_{z} r^{2} / 2 c\right)\right]_{m n}}{E_{n}-E_{m}}, \\
\hat{L}_{z}=-i \hbar\left(x \frac{\partial}{\partial y}-y \frac{\partial}{\partial x}\right) .
\end{gathered}
$$

In the absence of a magnetic field, the quantities $D_{x}, D_{y}$ are identically equal to zero. Using the properties of the matrix elements for the linear oscillators we obtain that in the case of elliptic (circular) dots $D_{x}=D_{y}=0$. Keeping the term which is quadratic in $\mathbf{q} \cdot \mathbf{r}$ in the expansion of the exponent $\exp (i \mathbf{q} \cdot \mathbf{r})$, we obtain a nonzero contribution but the corresponding spin-flip rate is smaller than the contribution $\Gamma_{1}$ by a factor of $(\beta / s)^{2}\left(\omega_{c} / \omega_{0}\right)^{2}<1$; here, $\omega_{c}=e B_{z} / m c$. It is also clear that in the case of irregular dots quantities $D_{x, y}$ are not equal to zero. The ratio of the corresponding rate and $\Gamma_{1}$ can be estimated as $\tau^{2}\left(m_{0} \beta a / \hbar\right)^{2}$, where $\tau$ is a dimensionless parameter which describes the deviation from ellipticity and $a$ is a dot size. Even when $\tau \simeq 1$ this ratio is of the order of unity for a typical dot size $a \approx 10^{3} \AA$. Therefore, for $\tau$ $\ll 1$ we can expect that the contribution, Eq. (10), is much smaller than $\Gamma_{1}$.

Note that, besides the term $\hat{\mathcal{H}}_{1}$ which is linear in the 2D momentum, the initial Hamiltonian also contains the term which is cubic in the momentum: (1/2) $\hat{\sigma}_{x}\left\{\hat{p}_{x}, \hat{p}_{y}^{2}\right\}_{+}$ $-(1 / 2) \hat{\sigma}_{y}\left\{\hat{p}_{y}, \hat{p}_{x}^{2}\right\}_{+}$. Again, in the presence of the orbital magnetic field we could get some contribution to the spinflip rate. To this end, we need to calculate the quantities $\widetilde{D}_{x}, \widetilde{D}_{y}$ obtained from $D_{x}, D_{y}$ by replacing the operator $\hat{L}_{z}$ $+\left(e B_{z} r^{2} / 2 c\right)$ by $(1 / 2)\left\{\hat{p}_{x}, \hat{p}_{y}^{2}\right\}_{+}$or $(1 / 2)\left\{\hat{p}_{y}, \hat{p}_{x}^{2}\right\}_{+}$. In the case of elliptic (circular) dots we obtain $\widetilde{D}_{x}=\widetilde{D}_{y}=0$ because of the symmetry.

\section{B. Direct spin-phonon coupling}

Using the standard presentation for the strain tensor in terms of the acoustic phonon modes, we calculate the matrix element of $\hat{\mathcal{H}}_{2}$ for the electron spin-flip transition between the Zeeman sublevels of orbital state $\Phi$ with emission of a phonon with momentum q:

$$
\begin{aligned}
M_{\uparrow, \downarrow}= & \frac{V_{0}}{4}\left(\frac{\hbar}{2 \rho \omega_{q}}\right)^{1 / 2}\left[q_{x} e_{y}+q_{y} e_{x}\right]\langle\Phi| \frac{1}{2}\left\{\left(\hat{p}_{x}\right.\right. \\
& \left.\left.+i \hat{p}_{y}\right), \exp (i \mathbf{q} \cdot \mathbf{r})\right\}_{+}|\Phi\rangle .
\end{aligned}
$$

For simplicity, here we set $\mathbf{B} \| z$. Similar expressions were obtained in Ref. 13 for a different problem. The total spinflip rate is given by the Fermi golden rule: 


$$
\begin{aligned}
\Gamma_{2}= & \frac{\pi \hbar V_{0}^{2}}{16 \rho g \mu_{B} B} \int \frac{d^{3} q}{(2 \pi)^{3}}\left(q_{x}^{2}+q_{y}^{2}\right) \mid\langle\Phi| \frac{1}{2}\left\{\left(\hat{p}_{x}\right.\right. \\
& \left.\left.+i \hat{p}_{y}\right), \exp (i \mathbf{q} \cdot \mathbf{r})\right\}\left._{+}|\Phi\rangle\right|^{2} \delta\left(\hbar s q-g \mu_{B} B\right) .
\end{aligned}
$$

The relevant phonon wavelength is much larger than the dot size, which allows for further simplifications. We concentrate on a circular dot with confining frequency $\omega_{0}$. For the orbital states with $n=0$ and $l=0, \pm 1$ (the ground and the first two excited states),

$$
\Gamma_{2}=\frac{V_{0}^{2}\left(g \mu_{B} B\right)^{5}}{240 \pi \rho s^{7} \hbar^{4}}\left[l+\frac{\omega_{c}}{2 \sqrt{\omega_{0}^{2}+\left(\omega_{c}^{2} / 4\right)}}(|l|+1) !\right]^{2} .
$$

The spin-flip rate produced by the term $\hat{\mathcal{H}}_{3}$ does not depend on the structure of the orbital state and is given by

$$
\Gamma_{3} \simeq \frac{\left(\mu_{B} \tilde{g} B\right)^{2}\left(\mu_{B} g B\right)^{3}}{\rho s_{t}^{5} \hbar^{4}}
$$

Let us now compare the rates $\Gamma_{1,2,3}$ obtained. All of them are proportional to the fifth power of energy splitting $g \mu_{B} B$, so that their ratio hardly depends on the magnetic field. First, we note that the ratio of $\Gamma_{3}$ and $\Gamma_{2}$ is of the order of $\left(\tilde{g} s_{t} / g V_{0}\right)^{2} \approx 7.8 \times 10^{-3} \ll 1$, so that $\Gamma_{2}$ is more important. The ratio of $\Gamma_{1}$ and $\Gamma_{2}$ is of the order of $\left(e h_{14} / m V_{0} / \hbar\right)^{2}\left[m \beta^{2} m s_{t}^{2} /\left(\hbar \omega_{0}\right)^{4}\right]$. For $\hbar \omega_{0} \simeq 1-10 \mathrm{~K}$ the ratio is of the order of $10^{6}-10^{2}$ and increases only for larger dots that have smaller $\omega_{0}$. Thus, we conclude that the admixture mechanism dominates.

\section{Two-phonon processes}

The calculated rate $\Gamma_{1}$ is small partly because of the small phonon density of the states at the scale of the Zeeman energy. On the other hand, for the case of the spin-flip transitions between the Zeeman levels of the usual impurity ${ }^{11}$ the two-phonon processes under some conditions may become more important than the single-phonon processes. At sufficiently small Zeeman splitting the contribution of the singlephonon processes is very small, and with increasing temperature the role of the processes when one phonon is absorbed and the other is emitted is increased. It is also true for the case of a quantum dot and here we give some formulas which describe the contribution of such two-phonon processes for GaAs quantum dots in several limiting cases. We also indicate the conditions under which these contributions can be important.

If we treat the interaction with the phonons in the second order, we obtain processes in which a phonon is scattered from state $\mathbf{p}$ to state $\mathbf{q}$ while the electron spin flips. The effective matrix element contains transitions to an excited orbital state with the emission or absorption of a phonon and then transitions back to the ground state with the absorption or emission of a phonon. The spin may flip either in the first or second transition. The matrix element is ${ }^{11}$

$$
\begin{aligned}
\left\langle V_{2}\right\rangle \sim & \left(\frac{\hbar}{\rho s \sqrt{p q}}\right)\left(e h_{14}\right)^{2}\left[N_{p}\left(N_{q}+1\right)\right]^{1 / 2} \\
& \times \sum_{a}\left\{\frac{\left[H_{p,-q}^{+}+H_{p,-q}^{-}\right]}{-\Delta_{a}-\hbar s q}+\frac{\left[H_{-q, p}^{+}+H_{-q, p}^{-}\right]}{-\Delta_{a}+\hbar s p}\right\}, \\
H_{p, q}^{ \pm} & =\left(\Psi_{0}^{+}, \exp (i \mathbf{p} \cdot \mathbf{r}) \Psi_{a}^{ \pm}\right)\left(\Psi_{a}^{ \pm}, \exp (i \mathbf{q} \cdot \mathbf{r}) \Psi_{0}^{-}\right),
\end{aligned}
$$

where $N_{p}$ is the Bose distribution function and $\Delta_{a}$ is the energy separation between the ground state whose wave function is $\Psi_{0}$ and the excited state whose wave function for spin up, say, is $\Psi_{a}^{+}$. We can neglect the Zeeman energy in the denominators since no Van Vleck cancellation occurs here. We consider again an interaction with piezophonons since deformation phonons become important at very high temperature (see below). For simplicity we consider here only the case when the magnetic field is perpendicular to the 2D plane and study the relaxation of the $S_{z}$ spin component.

As was shown in Ref. 4, there is a cancellation of the linear in the $\beta$ terms in the matrix elements of type $\left(\Psi_{0}^{+}, \exp (i \mathbf{p} \cdot \mathbf{r}) \Psi_{a}^{-}\right)$for the spin-flip transition between different orbital levels. This is a consequence of the fact that spin-orbit terms $\hat{\mathcal{H}}_{1}$ are linear in the lateral momentum operators $\hat{p}_{x, y}$. For that reason, quantities $H_{p, q}^{ \pm}$are proportional to the first power of $\beta$ only if one takes into account the Zeeman splitting in the electron spectrum. ${ }^{4}$ We consider the temperature in the interval $g \mu_{B} B \ll T \ll \hbar \omega_{0}$, where $\hbar \omega_{0}$ is the characteristic energy distance between the levels in the dot. Since $\hbar s p \simeq \hbar s q<T$, then we can neglect the phonon energies in the denominators while calculating the contribution to $\left\langle V_{2}\right\rangle$ proportional to the first power of $\beta$. It is apparent that the spin-flip rate has a different temperature dependence for the temperatures smaller and larger than $T_{0}$ $\simeq \sqrt{m s^{2} \hbar \omega_{0}}$. At this characteristic temperature the phonon wavelength is equal to the lateral dot size $\lambda$. For GaAs at $\hbar \omega_{0} \simeq 10 \mathrm{~K}$, the temperature $T_{0} \approx 1 \mathrm{~K}$. Let us give the estimate for the spin-flip rate in the case $T \ll T_{0}$, when $p \lambda, q \lambda$ $\ll 1$. Here the estimate for $H_{p, q}^{ \pm}$is $H_{p, q}^{ \pm} \simeq\left(\beta / \lambda \omega_{0}\right)$ $\times\left(g \mu_{B} B / \hbar \omega_{0}\right)(\lambda q)^{3}$. Then the relaxation rate is

$$
\begin{aligned}
\Gamma_{1}^{(2)}(T) & =(2 \pi / \hbar) \sum_{p, q}\left|\left\langle V_{2}\right\rangle\right|^{2} \delta\left[\hbar s(p-q)-g \mu_{B} B\right] \\
& \simeq \frac{\Lambda_{p}^{2}}{\hbar} \frac{s^{2}}{\beta^{2}} \frac{\left(g \mu_{B} B\right)^{2}\left(m s^{2}\right)^{5 / 2}}{\left(\hbar \omega_{0}\right)^{7 / 2}}\left(\frac{T}{T_{0}}\right)^{9}
\end{aligned}
$$

In performing the integral over $\mathbf{p}$, we have neglected $g \mu_{B} B$ in comparison to $\hbar s p$.

In the case $T \gg T_{0}$ the momentum components parallel to the plane are estimated as $q_{\|} \lambda \simeq 1$; otherwise, the matrix elements are exponentially small. As to the $q_{z}$ values, the contribution of the region $q_{z} \simeq T / \hbar s \gg q_{\|}$is much smaller than that where $q_{z} \simeq q_{\|} \ll T / \hbar s$. Such is the case even without regard for the fact that for the [100] orientation of the 2D plane the effective piezomodulus $A_{\mathbf{q} \alpha}$ introduced above has a smallness $q_{\|} / q_{z} \ll 1$. Thus, calculating the contribution from 
$q_{z} \simeq q_{\|} \ll T / \hbar s$ and taking into account that $N_{p}=T / \hbar s p \gg 1$, we obtain for the spin-flip rate in the case $T_{0} \ll T \ll \hbar \omega_{0}$

$$
\Gamma_{2}^{(2)}(T) \simeq \frac{\Lambda_{p}^{2}}{\hbar} \frac{s^{2}}{\beta^{2}} \frac{\left(g \mu_{B} B\right)^{2}\left(m s^{2}\right)^{5 / 2}}{\left(\hbar \omega_{0}\right)^{7 / 2}}\left(\frac{T}{T_{0}}\right)^{2} .
$$

The contribution from the deformation phonons is much smaller. In the case $T \gg T_{0}$ the characteristic $q_{z} \simeq T / \hbar s \gg q_{\|}$ $\simeq 1 / \lambda$; i.e., the deformation phonons are emitted almost perpendicular to the $2 \mathrm{D}$ plane. Then, for the deformation potential contribution to the spin-flip rate we obtain $\Gamma_{d} \simeq\left(\Lambda_{d}^{2} / \hbar\right)$ $\times\left(\beta^{2} / s^{2}\right)\left(g \mu_{B} B\right)^{2} T^{3} /\left(\hbar \omega_{0}\right)^{3} m s^{2}$, where $\Lambda_{d} \simeq(1 / 2 \pi)$ $\times\left(\Sigma^{2} m^{2} / \rho \hbar^{3} s\right)$ is the dimensionless constant which shows the strength of the electron interaction with deformation phonons. For GaAs, $\Lambda_{d} \approx 10^{-5}$. Even at $T \simeq \hbar \omega_{0}$ the value of $\Gamma_{d} / \Gamma_{2}^{(2)} \simeq\left(\hbar \omega_{0} / m s^{2}\right)^{5 / 2}\left(\Lambda_{d} \beta^{2} / \Lambda_{p} s^{2}\right)^{2}$ is much smaller than unity for any realistic $\hbar \omega_{0}$. For example, at $\hbar \omega_{0}=30 \mathrm{~K}$ this ratio is $\approx 0.03$.

Let us compare the two-phonon contribution $\Gamma_{2}^{(2)}$ with $\Gamma_{1} T / g \mu_{B} B$. We see that the two-phonon contribution $\Gamma_{2}^{(2)}$ prevails at sufficiently small Zeeman splittings: $g \mu_{B} B$ $<m s^{2}\left(\Lambda_{p} s^{2} / \beta^{2}\right)^{1 / 2}\left(T / T_{0}\right)^{1 / 2}$. Taking the maximal temperature $T \simeq \hbar \omega_{0}$, we obtain $g \mu_{B} B<\left[\left(\Lambda_{p} s^{2} / \beta^{2}\right)\right.$ $\left.\times \sqrt{m s^{2} / \hbar \omega_{0}}\right]^{1 / 2} T_{0}$. For $\hbar \omega_{0} \approx 10 \mathrm{~K}$ we see that this contribution is more important for magnetic fields smaller than approximately $0.4 \mathrm{~T}$ (where the estimate for the spin-flip time is of the order of ms). On the other hand, at $T \simeq T_{0}$ we obtain for the same $\hbar \omega_{0}$ that $g \mu_{B} B<0.03$ K (i.e., the twophonon contribution is more important for magnetic fields smaller than $\approx 1 \mathrm{kG}$ ). For these fields the characteristic spin-flip time is of the order of $1 \mathrm{~s}$; i.e., it is still long.

The general conclusion is that at sufficiently low temperatures (much smaller than $\hbar \omega_{0}$ ) the characteristic Zeeman splittings below which the two-phonon contribution to the spin-flip rate dominates are small and the corresponding spin-flip times are unusually long (see the estimates above).

\section{OTHER MECHANISMS OF THE SPIN FLIP}

Let us discuss briefly other mechanisms of the spin flip. The spin transitions between the Zeeman sublevels of the impurity state in semiconductors (mostly $\mathrm{Si}$ ) were extensively studied quite a long time ago. ${ }^{14,11}$ Except spin-orbit coupling, several other mechanisms were proposed, such as (1) modulation of the hyperfine coupling with nuclei by lattice vibrations, (2) the spin-spin interaction between the bound electron and the conduction electron in the leads, (3) the spin-current interaction, when the bound electron spin flip is caused by the fluctuating magnetic field of the conduction electrons, and (4) an exchange scattering process which flips the spins of both the conduction electron and the bound donor electron. Whereas the spin-orbit interaction strongly depends on the crystal symmetry and is different for Si and GaAs, the other mechanisms are quite general in nature and we can profit from the discussion in Refs. 14 and 11 .

Mechanism (4) requires an overlap of the wave functions of the electrons in the leads and in the dot. In the context of QD it is considered in Ref. 15. The corresponding rates are not intrinsic to the dot since they are proportional to the barrier transparencies. They can be tuned to arbitrary low values. References 11 and 14 have demonstrated that the spin-flip rates associated with mechanisms (2) and (3) are very small. As an example, we give the rate estimation for mechanism (3). The Bio-Savar formula relates the magnetic field and current fluctuations in the leads so that $\left\langle H^{2}\right\rangle_{\omega}$ $\simeq\left(1 / c^{2} a^{2}\right)\left\langle I^{2}\right\rangle_{\omega}, a$ being the characteristic distance between the electron in the dot and the electrons in the leads. Using the Nyquist formula for the correlator of the currents we estimate $\left\langle H^{2}\right\rangle_{\omega} \simeq\left(1 / c^{2} a^{2}\right) \hbar \omega \operatorname{coth}(\hbar \omega / 2 T)(1 / R), R$ being the typical resistance of the leads or the dot environment. Thus, the corresponding spin-flip relaxation rate is estimated as

$$
\Gamma_{4} \simeq \mu_{B}^{2}\left\langle H^{2}\right\rangle_{\omega} / \hbar^{2} \simeq \omega\left(\frac{\lambda_{c}}{a}\right)^{2} \frac{\hbar}{e^{2} R},
$$

where $\lambda_{c}=e^{2} / m_{0} c^{2} \approx 2.8 \times 10^{-13} \mathrm{~cm}$ is the classical electron radius, and $\hbar \omega=g \mu_{B} B$. The rate $\Gamma_{4}$ is proportional to the first power of Zeeman splitting so that it may formally compete with $\Gamma_{1}$ at sufficiently small splittings. However, this occurs at splittings that are so small that the corresponding rates are not observable. To give an example, we choose $R=1 \Omega$ and $\hbar \omega_{0}=1 \mathrm{~K}$, which corresponds to $a \simeq 1.15$ $\times 10^{-5} \mathrm{~cm}$. Then the rate $\Gamma_{4}$ would dominate if splitting $\hbar \omega \ll 2.5 \times 10^{-3} \mathrm{~K}$. This corresponds to the rates lower than $8 \times 10^{-4} \mathrm{~s}^{-1}$.

As to mechanism (1), i.e., modulation of the hyperfine coupling with nuclei by lattice vibrations, the relative strength of this mechanism and the spin-orbit interaction can be different for different materials. For example, in the case of $\mathrm{Si}$ where the spin-orbit interaction is much weaker than in $\mathrm{GaAs}$, the dominant mechanism of the spin flip for the case of the phonon-assisted transitions between the Zeeman levels of the usual impurity (the situation studied in Ref. 14) was found to be the modulation of the hyperfine coupling with nuclei by lattice vibrations. In the case of GaAs, however, our conclusion is that the dominant mechanism is the admixture mechanism of the spin-orbit interaction. This conclusion was reached for the first time in Ref. 16, where the calculations used essentially followed those in Ref. 14. Here we give the result obtained in Ref. 16 for the rate due to the modulation of the hyperfine coupling with nuclei by lattice vibrations

$$
\Gamma_{h} \simeq\left(g \mu_{B} B\right)^{3} \gamma^{2} \omega_{N}^{2} / \hbar^{2} s^{5} \rho,
$$

where $\omega_{N} \simeq\left(v_{0} A^{2} / a^{2} z_{0}\right)^{1 / 2} / \hbar$ is the electron spin precession frequency in the random field of unpolarized nuclei, $v_{0}$ is the unit cell volume, $A$ the hyperfine interaction constant, $a$ the dot lateral size, and $z_{0}$ the electron wave function extension in the $z$ direction. Finally, $\gamma \simeq(1 / m)(d m / d \Delta)$ is the change in effective mass $m$ with dilation; see also Ref. 14. For GaAs QD's with $a \approx 10^{3} \AA$ and $z_{0} \approx 10^{2} \AA, \omega_{N}$ can reach $\simeq 10^{8} \mathrm{~s}^{-1}$. Let us compare the spin-flip rate, Eq. (20), with $\Gamma_{1}$ (this comparison was done earlier in Ref. 16). Even taken for $\gamma \simeq 50$ (see also Ref. 14) we can easily see that $\Gamma_{h}$ will compete with $\Gamma_{1}$ at the Zeeman splitting $\simeq \gamma \omega_{N}\left(\hbar \omega_{0}\right)^{2} /$ $\beta e h_{14}$ which is so small that the corresponding rate is not observable. For example, for $\hbar \omega_{0}=10 \mathrm{~K}$ the splitting is of 
the order of $10^{-5} \mathrm{~K}$. Therefore, the admixture mechanism of the spin-orbit interaction is the dominant one.

It should be noted that in this work we have not considered the electron spin relaxation mechanism which is through the hyperfine interaction related to the internal nuclear dynamics. The latter is due to the dipole-dipole interaction between the nuclei which does not conserve the total nuclear spin. ${ }^{17}$ This mechanism might be important at low magnetic fields. However, this problem is not simple and needs a separate investigation.

Finally, we mention the experimental studies of spin relaxation in $n$-type GaAs quantum dots. Such an experiment has been recently carried out. ${ }^{18}$ The nonequilibrium tunneling current through excited states in an $\mathrm{AlGaAs} / \mathrm{GaAs}$ quantum dot was studied using a pulse-excitation technique which measures the energy relaxation time from the excited state to the ground state. Some excited states showed a relaxation time which was much longer than a few $\mu$ s, while the other showed time much shorter than a few ns. This great difference in relaxation times was ascribed to the fact that some inelastic transitions are accompanied by the spin flip. For these transitions the relaxation time was so long that the method used in the above-mentioned paper only allowed us to give some estimation (much longer than a few $\mu$ s). Though the transitions studied by Fujisawa et al. could in general involve the spin-flip transitions between the states with different orbital structures (this situation was considered in our previous paper ${ }^{4}$ ), the experimental data confirm the general statement that the spin-flip processes in $n$-type quantum dots can be really slow.

In conclusion, we have calculated the rates for the phonon-assisted spin-flip transitions between the Zeeman sublevels in a quantum dot for all possible mechanisms and shown that the admixture mechanism of the spin-orbit interaction is a dominant one. The corresponding spin-flip rate $\Gamma_{1}$ [see Eqs. (7) and (8)] exhibits a strong dependence on Zeeman energy and at small magnetic fields takes very low values (up to seconds).

This work was part of the research program of the "Stichting voor Fundamenteel Onderzoek der Materie (FOM)." We acknowledge the support of the Netherlands Organization for Scientific Research (NWO) in the framework of the DutchRussian collaboration and NEDO Project No. NTDP-98. We are grateful to L. P. Kouwenhoven, T. H. Oosterkamp, G. E. W. Bauer, T. Fujisawa, Y. Tokura, Y. Hirayama, and D. Loss for useful discussions.
*Permanent address: Institute of Microelectronics Technology, Russian Academy of Sciences, 142432, Chernogolovka, Moscow District, Russia.

${ }^{1}$ R. C. Ashoori, Nature (London) 379, 413 (1996).

${ }^{2}$ L. P. Kouwenhoven, C. M. Marcus, P. L. McEuen, S. Tarucha, R. M. Westervelt, and N. S. Wingreen, in Mesoscopic Electron Transport, edited by L. L. Sohn, G. Schon, and L. P. Kouwenhoven (Kluwer, Dordrecht, 1997), p. 345.

${ }^{3}$ D. Loss and D. P. DiVincenzo, Phys. Rev. A 57, 120 (1998).

${ }^{4}$ A. V. Khaetskii and Yu. V. Nazarov, Phys. Rev. B 61, 12639 (2000).

${ }^{5}$ G. E. Pikus and A. N. Titkov, Optical Orientation (NorthHolland, Amsterdam, 1984), p. 73.

${ }^{6}$ M. I. D'yakonov and V. Yu. Kachorovskii, Fiz. Tech. Poluprovodn. 20, 178 (1986) [Sov. Phys. Semicond. 20, 110 (1986)]

${ }^{7}$ M. I. D'yakonov et al., Zh. Éksp. Teor. Fiz. 90, 1123 (1986) [Sov. Phys. JETP 63, 655 (1986)].

${ }^{8}$ C. Kittel, Quantum Theory of Solids (Wiley, New York, 1967), Chap. 14.
${ }^{9}$ V. F. Gantmakher and Y. B. Levinson, Carrier Scattering in Metals and Semiconductors (North-Holland, Amsterdam, 1987), Chaps. 3 and 4.

${ }^{10}$ J. H. Van Vleck, Phys. Rev. 57, 426 (1940).

${ }^{11}$ E. Abrahams, Phys. Rev. 107, 491 (1957).

${ }^{12}$ L. D. Landau and E. M. Lifshitz, Quantum Mechanics (Pergamon, New York, 1963).

${ }^{13}$ D. Frenkel, Phys. Rev. B 43, 14228 (1991).

${ }^{14}$ D. Pines, J. Bardeen, and C. P. Slichter, Phys. Rev. 106, 489 (1957).

${ }^{15}$ A. Kaminski, Yu. V. Nazarov, and L. I. Glazman, Phys. Rev. Lett. 83, 384 (1999); A. Kaminski, Yu. V Nazarov, and L. I. Glazman, Phys. Rev. B 62, 8154 (2000).

${ }^{16}$ A. V. Khaetskii, in Proceedings of the International Conference PASPS 2000, Sendai, 2000 [Photonics Spectra 10, 27 (2001)].

${ }^{17}$ A. Abragam, The Principles of Nuclear Magnetism (Oxford University Press, New York, 1961).

${ }^{18}$ T. Fujisawa, Y. Tokura, and Y. Hirayama, Phys. Rev. B 63, 081304(R) (2001). 